\title{
Perfil clínico e epidemiológico dos pacientes portadores de hanseníase em uma unidade de referência no estado do Pará
}

\author{
Clinical and epidemiological profile of patients with hanseniasis in a reference unit in the \\ state of Pará
}
Perfil clínico y epidemiológico dos pacientes portadores de hanseníase em uma unidade de referência no estado do Pará

Mariana do Socorro Maciel Quaresma1, Larissa da Silva Costa Souza1, Felype Bastos Marques da Silva ${ }^{1}$, Carla Daniele Nascimento Pontes ${ }^{1 *}$, Yuri José Almeida Da Silva ${ }^{2}$.

\begin{abstract}
RESUMO
Objetivo: Avaliar o perfil clínico e epidemiológico dos pacientes portadores de Hanseníase em uma Unidade de referência no Estado do Pará. Método: Trata-se de uma pesquisa quantitativa do tipo descritiva, retrospectiva e transversal, no período de janeiro de 2013 a janeiro de 2015. Resultados: A maioria dos casos de hanseníase ocorreu entre a faixa etária de 30 a 39 anos, sendo mais prevalente no gênero masculino. As formas clínicas mais frequentes foram a Dimorfa $(55,5 \%)$ e Virchowiana (23,5\%). As formas multibacilares corresponderam a $79 \%$. E no tocante a incapacidade física, houve tendência significante para ausência de incapacidade com $53 \%$ dos pacientes quanto a terapia medicamentosa proposta, obteve-se completa adesão de (99,5\%) dos pacientes. Conclusão: o perfil clínico e epidemiológico dos pacientes com hanseníase atendidos pela Unidade de Referência, em geral é caracterizado por uma predominância do gênero masculino no período de vida adulto, tendo como maior procedência a região metropolitana de Belém. A forma Multibacilar foi predominante, possuindo maior prevalência da classificação clínica Dimorfa. Neste estudo mais de $50 \%$ dos pacientes não apresentaram qualquer sinal de incapacidade e a maioria destes foram encaminhados à unidade de referência através da atenção primaria de saúde. Ocorreu alta adesão ao tratamento, podendo ser reflexo da implantação de políticas de educação a respeito da doença para a comunidade, do trabalho sério e dedicado prestado por essa unidade de referência que preza pelo seguimento adequado deste paciente a fim de evitar a progressão das incapacidades funcionais, assegurando a melhor qualidade de vida do mesmo.
\end{abstract}

Palavras-Chave: Hanseníase, Epidemiologia, Atenção básica.

\begin{abstract}
Objective: To evaluate the clinical and epidemiological profile of patients with leprosy in a reference unit in the state of Pará. Method: This is a descriptive, retrospective and cross-sectional quantitative survey from January 2013 to January 2015 Results: Most cases of leprosy occurred between the ages of 30 and 39 years, being more prevalent in the male gender. The most frequent clinical forms were Dimorfa $(55.5 \%)$ and Virchowiana (23.5\%). The multibacillary forms corresponded to $79 \%$. Regarding the physical disability, there was a significant tendency for the absence of incapacity with $53 \%$ of the patients. As for the proposed drug therapy, it was obtained complete adherence of $(99.5 \%)$ patients. Conclusion: the clinical and epidemiological profile of patients with leprosy assisted by the Reference Unit is generally characterized by a predominance of the male gender in the adult life, with the Belém metropolitan region as its main source. The Multibacillary
\end{abstract}

${ }^{1}$ Centro Universitário do Pará, Belém-PA. * E-mail: pontes4@live.com

2Universidade Federal do Pará, Belém-PA

SUBMETIDO EM: 10/2018

ACEITO EM: 11/2018

PUBLICADO EM: 01/2019

REAS/EJCH | Vol.Sup.18 | e269 | DOI: https://doi.org/10.25248/reas.e269.2019 Página 1 de 10 
form was predominant, higher prevalence of the Dimorfa clinical classification. In this study, more than $50 \%$ of the patients did not present any sign of disability and most of them were referred to the referral unit through primary health care. There was a high adherence to treatment, which may be a reflection of the implementation of education policies regarding the disease to the community, of the serious and dedicated work provided by this reference unit that values the proper follow-up of this patient in order to avoid the progression of functional disabilities, ensuring the best quality of life.

Keywords: Leprosy, Epidemiology, Primary Care.

\section{RESUMEN}

Objetivo: Evaluar el perfil clínico y epidemiológico de los pacientes portadores de Hansenias en una Unidad de referencia en el Estado de Pará. Método: Se trata de una investigación cuantitativa del tipo descriptivo, retrospectivo y transversal, en el período de enero de 2013 a enero de 2015. Resultados: La mayoría de los casos de hanseniasis ocurrieron entre el grupo de edad de 30 a 39 años, siendo más prevalente en el género masculino. Las formas clínicas más frecuentes fueron la Dimorfa (55,5\%) y Virchowiana (23,5\%). Las formas multibacilares correspondieron al $79 \%$. En cuanto a la incapacidad física, hubo una tendencia significante para la ausencia de incapacidad con el $53 \%$ de los pacientes En cuanto a la terapia medicamentosa propuesta, se obtuvo una completa adhesión de $(99,5 \%)$ de los pacientes. Conclusión: el perfil clínico y epidemiológico de los pacientes con hanseniasis atendidos por la Unidad de Referencia, en general es caracterizado por una predominancia del género masculino en el período de vida adulto, teniendo como mayor procedencia la región metropolitana de Belém. La forma Multibacilar fue predominante, mayor prevalencia de la clasificación clínica de Dimorfa. En este estudio más del 50\% de los pacientes no presentaron ningún signo de incapacidad y la mayoría de éstos fueron encaminados a la unidad de referencia a través de la atención primaria de salud. Se ha producido una alta adhesión al tratamiento, pudiendo ser reflejo de la implantación de políticas de educación acerca de la enfermedad para la comunidad, del trabajo serio y dedicado prestado por esa unidad de referencia que preca por el seguimiento adecuado de este paciente a fin de evitar la progresión de las incapacidades funcionales, asegurando la mejor calidad de vida del mismo.

Palabras clave: Hansenias, Epidemiología, Atención Básica.

\section{INTRODUÇÃO}

O Brasil, é considerado um dos países com um dos maiores índices de casos de hanseníase (BRASIL 2010). Pode-se considerar que o Brasil esteja nessa posição pela sua grande extensão territorial, que dificulta os procedimentos de controle a doença; a falta de informação da população sobre a forma de contágio e tratamento; e a estigmatização histórica que a hanseníase traz consigo (CUNHA 2002).

Atualmente, a doença prevalece em alguns países do hemisfério sul, em áreas tropicais e subtropicais, embora já tenha sido endêmica na Europa, Índia, Brasil e Madagascar, detêm $90 \%$ dos casos relatados. A Organização Mundial da Saúde (OMS), apresenta como meta que todos os países atinjam prevalência menor que 1 caso/10.000 habitantes, o chamado projeto de eliminação da hanseníase (EIDT 2004).

A hanseníase é conceituada como uma doença infectocontagiosa, causada pelo Mycobacterium leprae, atingindo preferencialmente pele e/ou nervos periféricos. A doença pode atingir praticamente todos os órgãos e sistemas, exceto o sistema nervoso central. Evolui de forma crônica, podendo apresentar períodos de agudização denominados reações (CUNHA et al, 2007).

O Mycobacterium leprae ou bacilo de Hansen é uma bactéria em forma de bastão recurvado, trata-se de um bacilo álcool-ácido resistente (BAAR), é um parasito intracelular obrigatório, de crescimento lento, que 
apresenta afinidade por células cutâneas e por células dos nervos periféricos (células de Schwann) (BRASIL 2002).

O homem é reconhecido como a única fonte de infecção, embora tenham sido identificados animais naturalmente infectados como o tatu, o macaco mangabei e o chimpanzé (BRASIL 2002).

A transmissão é prevalentemente pelas vias aéreas superiores (BRASIL 2010). É transmitida principalmente por meio do convívio com os doentes do tipo virchowiano ou dimorfo que ainda não foram diagnosticados e tratados (CUNHA et al, 2007). Esses indivíduos são capazes de eliminar grande quantidade de bacilos para o meio exterior, carga bacilar de cerca de 10.000.000 de BAAR presentes na mucosa nasal (BRASIL 2002). Quando o paciente toma a primeira dose da PQT (Poliquimioterapia - Conjunto de medicamentos para tratamento da Hanseníase composto pela Rifampicina, Dapsona e Clofazimina) /OMS mais de $99 \%$ dos bacilos se tornam inviáveis e perdem a capacidade de infectar outras pessoas (BRASIL 2008).

O diagnóstico da Hanseníase é essencialmente clínico, tendo como base os sinais e sintomas e história epidemiológica do paciente (anamnese- tempo de manifestação, ocorrência de casos na família e percepção de parestesias e exame clínico- dermatológico, neurológico e avaliação oftalmológica). Em se tratando de uma doença contagiosa, granulomatosa, de evolução insidiosa (lenta) e de grande potencial incapacitante, o diagnóstico precoce deve ser o primeiro objetivo das ações de controle da Hanseníase. Na prática do dia a dia isso só é possível a partir das manifestações clínicas da doença. Outras provas complementares são os exames de laboratório - baciloscopia e histopatologia e a prova da histamina (BRASIL 2002).

Do ponto de vista operacional os casos devem ser classificados em Paucibacilares (PB), até cinco lesões de pele sem infiltração, e Multibacilares (MB), mais de cinco lesões independentemente de infiltração, ou até 5 cinco lesões, desde que estas sejam infiltradas. A classificação operacional proposta pela Organização Mundial de Saúde (OMS) visa facilitar o tratamento por parte dos profissionais que tenham dificuldade em classificar os casos de Hanseníase (AZULAY 2017).

A classificação proposta por Ridley e Jopliing em 1966, que reconhece seis formas de apresentação da Hanseníase baseando-se em características clínicas, histológicas e imunológicas: I (indeterminada), TT (tuberculoid-tuberculoid), DT (dimorfa-tuberculoid), DD (dimorfa-dimorfa), DV (dimorfa-virchowiana) e VV (virchowiana-vichorwiana). Classificação muito utilizada pelos patologistas, mas sem muito valor prático (BRASIL 2008).

Todos os casos diagnosticados devem ser notificados ao Sistema de Informação de Agravo de Notificação - SINAN e iniciarem imediatamente o tratamento específico para Hanseníase. O tratamento atual adotado pelo Ministério da Saúde é o mesmo adotado pela OMS. Ele se baseia no esquema de poliquimioterapia (PQT) em que múltiplas drogas são utilizadas no sentido de evitar a resistência bacteriana. Para a indicação do tratamento adequado, utiliza-se a classificação operacional, a qual é dividida em dois grupos: paucibacilar com tratamento de duração de 6 doses mensais supervisionadas em até 9 meses e multibacilar com tratamento de duração de 12 doses mensais supervisionadas em até 18 meses (CUNHA et al, 2007).

No esquema de tratamento paucibacilar as drogas utilizadas são a rifampicina (600 mg VO + dapsona $100 \mathrm{mg} \mathrm{VO}$, doses supervisionadas) e dapsona do $2^{\circ}$ aos $28^{\circ}$ dias (100 mg VO, dose diária, autoadministrada). Já no tratamento multibacilar, além das drogas anteriores é adicionado ao esquema uma terceira droga, a clofazimina (300 mg VO, dose mensal supervisionada + $50 \mathrm{mg} \mathrm{VO}$, dose diária, autoadministrada) (CUNHA et al, 2007).

\section{METODOLOGIA}

Foi realizada uma pesquisa quantitativa do tipo descritiva, retrospectivo, transversal, visando à análise do perfil clínico epidemiológico dos pacientes estudados. A população escolhida para estudo foi constituída de 
indivíduos de ambos os gêneros, com 15 anos ou mais, com diagnóstico confirmado de Hanseníase, matriculados na Unidade de Referência Especializada Dr. Marcello Candia. Foram analisadas as informações contidas nos prontuários de 200 pacientes cadastrados na Unidade de referência especializada Dr. Marcello Candia no município de Marituba-PA no período de janeiro de 2013 a janeiro de 2015. O número de prontuários foi definido em visita ao município de Marituba - PA, levando-se em consideração os seguintes critérios, de inclusão estar dentro da faixa etária pré-determinada, serem matriculados na Unidade de Referência especializada Dr. Marcello Candia, terem diagnósticos confirmado de hanseníase. Foram excluídos os prontuários que não estiveram dentro da faixa etária pré-estabelecida, que não eram da unidade de referência ou sem diagnóstico definitivo de Hanseníase.

Para realização da pesquisa o projeto foi aprovado no Comitê de Ética e Pesquisa do CESUPA conforme parecer de número 1.396.928. Foram utilizados como instrumentos os prontuários de atendimento dos pacientes para analisar faixa etária, gênero, procedência e classificação clínica. A análise estatística dos dados foi feita através de métodos estatísticos descritivos e inferenciais. As variáveis quantitativas foram apresentadas por medidas de tendência central e de variação. As variáveis qualitativas foram apresentadas por distribuições de frequências absolutas e relativas. A comparação entre as variáveis qualitativas foi realizada pelo teste do Qui-quadrado14. Foi previamente fixado o nível de significância alfa $=0.05$ para rejeição da hipótese de nulidade. O processamento estatístico foi realizado nos softwares GrafTable versão 2.0 e BioEstat versão 5.3 .

\section{RESULTADOS}

O presente estudo foi analisado dados de 200 pacientes com hanseníase com idade entre 15 e 99 anos, a média da idade foi 44 anos com desvio padrão de 18 anos. A maioria dos pacientes era do gênero masculino $57 \%$ ( $p$-valor $=0.0477^{\star}$ ), a faixa etária predominante ficou entre 30 a 39 anos $22 \%$ ( $p$-valor $<0.0001^{*}$ ) seguida de $60-79$ anos com $21 \%$ e $40-49$ anos com $18,5 \%$ do total de pacientes estudados. Quanto ao local de moradia, a maioria era da Região Metropolitana de Belém com 58,5\% ( $p$-valor $<0.0001^{*}$ ), seguido do Nordeste do Pará com 12,5\%. As manifestações Clínicas da Hanseníase apresentaram as seguintes características com tendência estatisticamente significante: Multibacilar $79 \%$ ( $p$-valor $<0.0001^{*}$ ) e Dimorfo $55,5 \%$ ( $p$-valor $<0.0001^{*}$ ) (Tabela 1).

Tabela 1 - Caracterização de acordo com a classificação da OMS, classificação de Madri e adesão ao tratamento em pacientes portadores de hanseníase em uma unidade de referência no Estado do Pará, ano 2016.

\begin{tabular}{lccc}
\hline \multirow{2}{*}{ Característica Clínica } & \multicolumn{3}{c}{ Pacientes } \\
\cline { 2 - 4 } & $\mathbf{n}$ & $\%$ & p-valor \\
\hline Classificação OMS & & & $<0,0001^{*}$ \\
\hline Multibacilar & 158 & 79 & \\
Paucibacilar & 42 & 21 & \\
\hline Formas Clínicas de Madrid & & & $<0,0001^{*}$ \\
\hline Tuberculoide & 26 & 13 & \\
Virchowiana & 47 & 21 & \\
Dimorfa & 111 & 55,5 & \\
Intermediária & 16 & 8 & \\
\hline Concluiu o Tratamento & \multicolumn{3}{c}{} \\
\hline Sim & 13 & 100 & \\
Não & 13 & 100 & \\
\hline
\end{tabular}

Fonte: Protocolo de pesquisa, ${ }^{*}$ Qui-quadrado de tendência. 
A distribuição dos Estados Reacionais mostrou que as seguintes características com tendência estatisticamente significante: Reação ausente $65 \%$ ( $p$-valor $<0.0001^{*}$ ). Entre os pacientes que tiveram reação hansênica, destacam-se: Tipo $1 \mathrm{com} 64,3 \%$ ( $p$-valor $<0.0001^{*}$ ) e estão em tratamento $77,1 \%$ ( $p$-valor $<0.0001^{*}$ ) (Tabela 2).

Tabela 2 - Caracterização dos estados reacionais e forma de ingresso dos pacientes portadores de hanseníase em uma unidade de referência no Estado do Pará, ano 2016.

\begin{tabular}{lccc}
\hline \multirow{2}{*}{ Estados Reacionais } & \multicolumn{3}{c}{ Pacientes } \\
\cline { 2 - 4 } & $\mathbf{n}$ & $\%$ & p-valor \\
\hline Estados reacionais & & & $<0,0001^{*}$ \\
\hline Presente & 70 & 35 & \\
Ausente & 130 & 65 & \\
\hline Tipos de Reação & & & $<0,0001^{*}$ \\
\hline Tipo 1 & 45 & 64,3 & \\
Tipo 2 & 12 & 17,1 & \\
Mista & 13 & 18,6 & \\
\hline Tratamento da Reação & & & $<0,0001^{*}$ \\
\hline$\quad$ Sim & 54 & 77 & \\
$\quad$ Não & 16 & 22,9 & \\
${ }^{*}$ Qui-quadrado de tendência & & & \\
Fonte: Protocolo de pesquisa. & & &
\end{tabular}

A distribuição da forma de ingresso demonstrou o predomínio da forma Encaminhamento com 90\%, com tendência estatisticamente significante ( $p$-valor $\left.<0.0001^{\star}\right)$.

No tocante a incapacidade houve tendência estatisticamente significante para: Ausência de incapacidade com 53\% (p-valor $\left.<0.0001^{*}\right)$ e Grau 0 com 53\% (p-valor $\left.<0.0001^{*}\right)$.

A avaliação da associação entre o grau de incapacidade e o gênero mostrou que os graus que indicam maior incapacidade apresentam maior frequência no gênero masculino: Grau I (32.5\%) e Grau II (25.4\%) quando comparados com o feminino Grau I (17,4\%) e Grau II (16,3\%), por isso o p-valor $=0.0030^{*}$.

Quanto à avaliação da associação entre o grau de incapacidade e a faixa etária do paciente mostrou que não existe real diferenciação, por isso o $p$-valor $=0.1448$ não é altamente significante.

Já na avaliação da associação entre o grau de incapacidade e a classificação da OMS mostrou que no tipo Multibacilar os graus que indicam maior incapacidade apresentam maiores proporções: Grau I (31\%) e Grau II (25,9\%) quando comparado com o Paucibacilar (7,1\% e 4,8\%, respectivamente), por isso o p-valor $=0.0030^{*}$ (Gráfico 1). 
Gráfico 1 - Correspondência entre a Classificação Operacional de acordo com a OMS e o Grau de incapacidade dos pacientes portadores de hanseníase em uma unidade de referência no Estado do Pará, ano 2016.

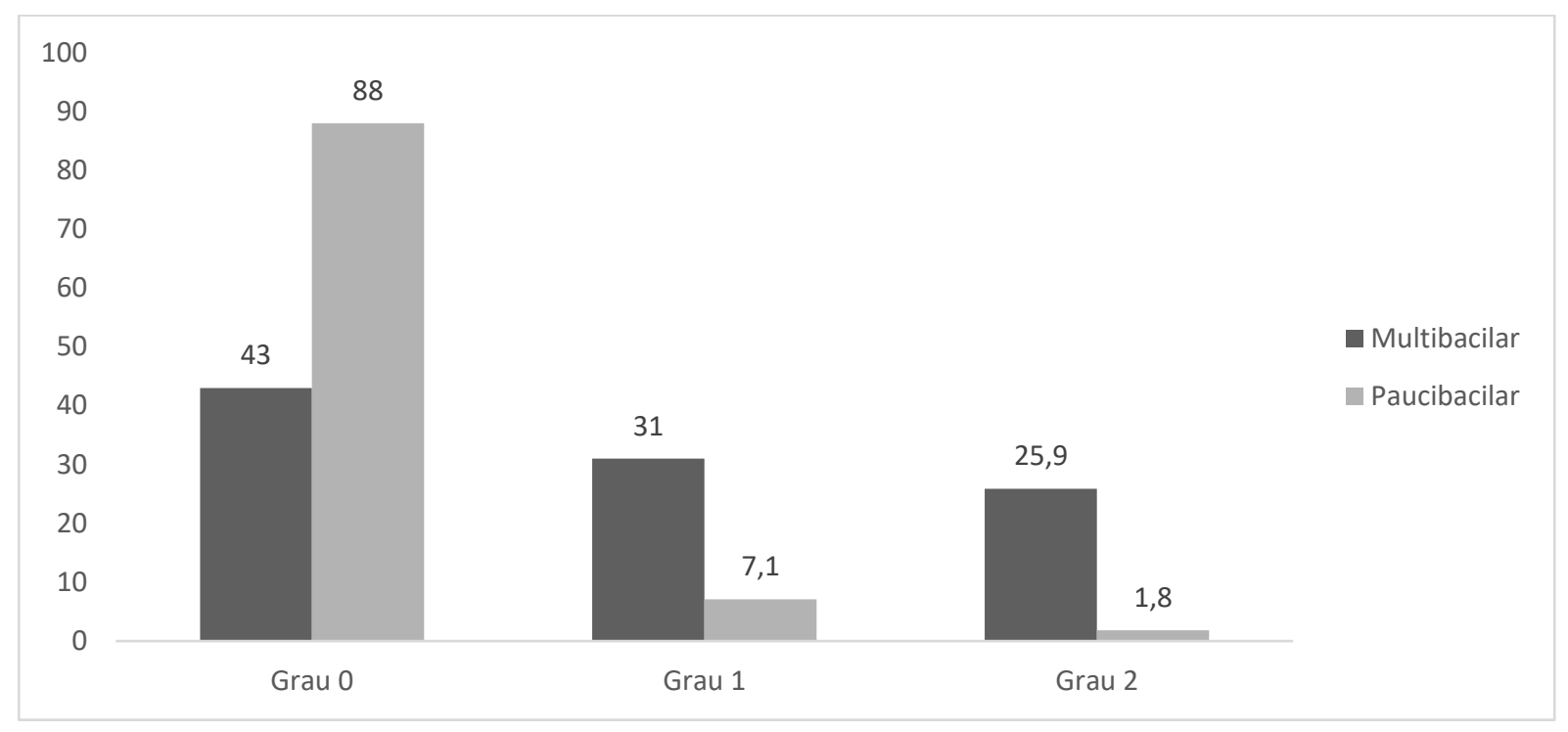

Fonte: Protocolo de Pesquisa.

E na avaliação da associação entre o grau de incapacidade e a classificação de Madri mostrou que o tipo Tuberculóide apresentam menos incapacidade Grau $0(84,6 \%)$ e o tipo Virchowiana apresenta $(29,8 \%)$ no Grau II, por isso o p-valor $<0.0001^{*}$ é altamente significante (Gráfico 2).

Gráfico 2 - Correspondência entre a Forma Clínica segundo a classificação de Madri e o Grau de incapacidade dos pacientes portadores de hanseníase em uma unidade de referência no Estado do Pará, ano 2016.

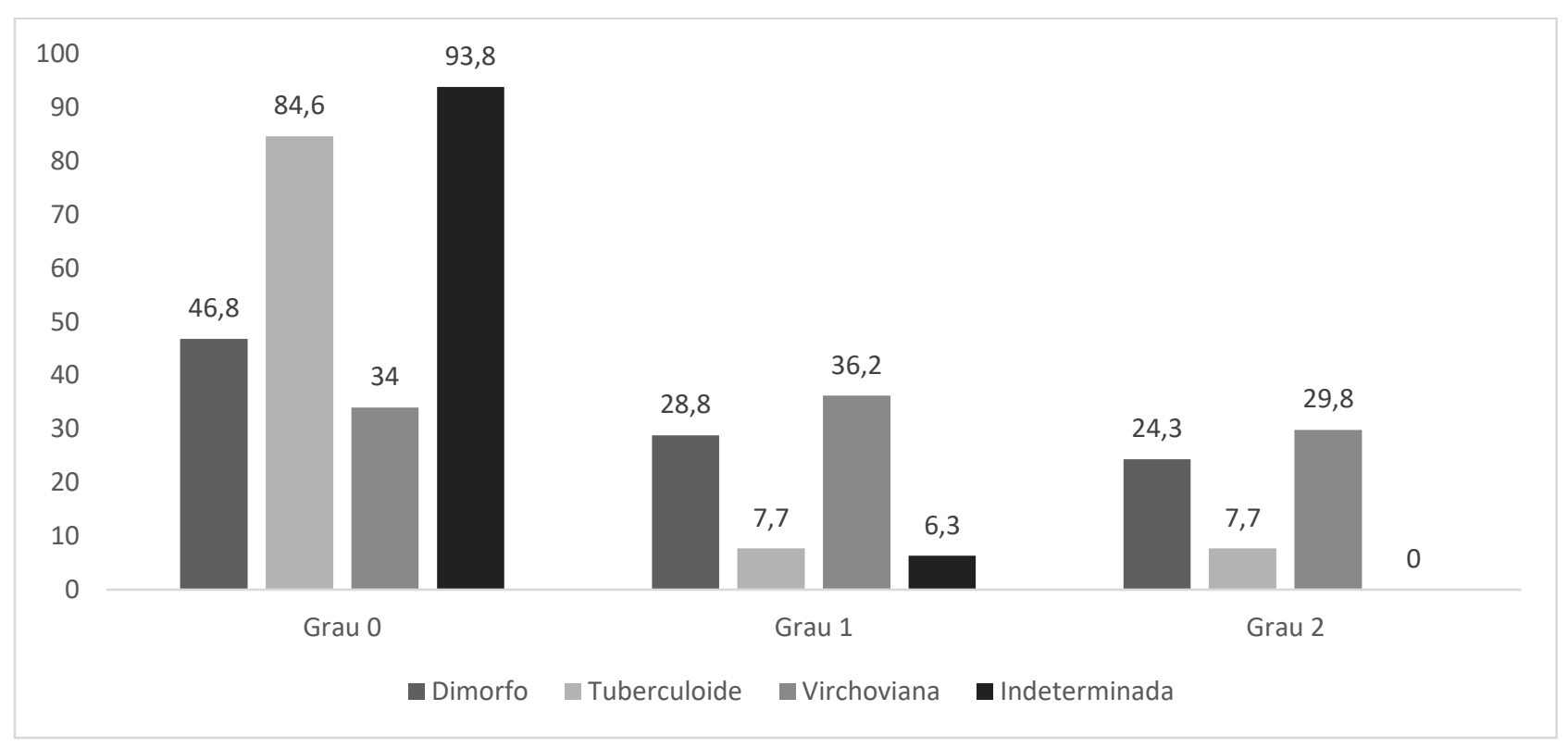

Fonte: Protocolo de pesquisa. 


\section{DISCUSSÃO}

O presente estudo permitiu identificar o comportamento da hanseníase em seus aspectos clínicos e epidemiológicos no município de Marituba - PA entre os anos considerados. Ao longo dos anos, notaram-se altas taxas de incidência e discretas flutuações entre os anos, justificadas pela dificuldade de controle da doença na região norte.

Neste trabalho observou-se maior prevalência no gênero masculino $(57 \%)$ concordando com o estudo de Batista e colaboradores que observaram que $(50,8 \%)$ dos seus pacientes pertenciam ao gênero masculino. Este resultado poderia traduzir uma maior oportunidade de contato social entre os homens, além de um exame clínico menos cuidadoso em mulheres. No entanto a literatura apresenta resultados divergentes. Segundo Lana e colaboradores 2013 informaram o encontro de $(55,3 \%)$ de casos entre as mulheres em consonância com o estudo de Andrade e Bonfim 2008, em que o gênero feminino foi o mais prevalente $(57,4 \%)$ e este resultado reflete o maior cuidado e atenção do gênero feminino quanto à procura aos serviços de saúde através da realização com maior frequência de consultas médicas, refletindo em um maior grau de orientação, por parte das mulheres, a respeito da patologia, formas de contágio e diagnóstico precoce. Desta forma, portanto, as mulheres teriam mais oportunidade de diagnóstico do que os homens. Entretanto Bakker em 2005 relatou o encontro de distribuição semelhante da doença entre os gêneros.

Durante o período analisado a faixa etária predominante ficou entre 30 a 39 anos (22\%), outros estudos corroboram esses achados, o mesmo foi visto em outros estudos demostrando que a população de adultos jovens é a mais acometida e demonstram que a doença predomina na faixa etária economicamente ativa e caracteriza a hanseníase como uma doença de adultos e adultos jovens, ocorrendo grande impacto socioeconômico. Sendo assim faz-se necessário ficar atento para o diagnóstico e tratamento precoce da hanseníase, pois quando o diagnóstico é realizado em uma faixa etária mais avançada, o paciente estará sujeito a evoluir de forma crônica com possível instalação de complicações funcionais (BERNARDES et al, 2009; LIMA et al, 2009).

Com relação à Classificação da OMS, houve a predominância da forma Multibacilar (79\%) superando a forma Paucibacilar que totalizou (21\%) dos casos. Concordando com o estudo de Aquino e Almeida 2000 que encontrou uma maior incidência de pacientes com hanseníase classificados como Multibacilares sobre os Paucibacilares no estado do Maranhão. Estes fatos fazem concordância com o estudo de pacientes com Hanseníase realizado na cidade de Botucatu - SP, onde Lastoria e Abreu 2012 encontrou maior dominância da forma Multibacilar. Este fato também foi demonstrado em um estudo realizado na cidade de Uberaba (MG), no período de 2000 a 2006 (BRASIL 2001). Esses achados são de extrema importância, uma vez que esta classe operacional é a principal fonte de transmissão da doença já que as mesmas apresentam elevada carga bacilar na derme e em mucosas, podendo ocorrer eliminação dos bacilos no meio exterior (IMBIRIBA et al 2008).

No que diz respeito às Formas Clínicas segundo a classificação de Madri, o presente estudo encontrou maior prevalência da forma Dimorfa (55,5\%), seguida pela forma Virchowiana em $(23,5 \%)$ dos pacientes e em terceiro lugar encontra-se a forma Tuberculóide (13\%), esta última, por sua vez, mais freqüente nas faixas etárias mais jovens, deixando em último lugar, a forma Indeterminada com (8\%) dos casos. Os resultados são compatíveis com o estudo de Gomes e colaboradores 2005, com predomínio da forma clínica Dimorfa $(54,6 \%)$, e apenas $5,8 \%$ deles, a forma Indeterminada. Divergindo do estudo de Batista e colaboradores que observaram um predomínio da forma Tuberculoide $(49,6 \%)$, Dimorfa $(21,3 \%)$ e Virchowiana $(15,4 \%)$. Estes achados demonstram atraso no diagnóstico nas unidades primárias de saúde, possibilitando a evolução das formas clínicas desta patologia e ocasionando maiores incapacidades funcionais. Segundo Barreto e colaboradores 2007 dar a classificação do paciente hansênico e forma clínica correta, nem sempre é tão simples.

Os Estados Reacionais foram identificados em $35 \%$ dos pacientes, sendo a reação Tipo 1 mais prevalente (64,3\%), seguido pela forma Mista (18,6\%) e com (18,6\%) a reação Tipo 2. Em consonância com o estudo de 
Silva e colaboradores 2008, a reação tipo 1 foi a mais freqüente entre os pacientes avaliados. Por outro lado, no estudo de Liendhardt e Fine 1994, da mesma forma que no presente estudo observou-se $(47,5 \%)$ de reações do tipo 1. Os Estados Reacionais estiveram presentes em todas as formas clínicas, porém com maior predomínio na classe Multibacilar. De acordo com Manandhar e colaboradores 1999 e Saunderson e colaboradores 2000 nas formas Multibacilares, existem fatores de risco importantes para o desenvolvimento principalmente da reação tipo 2. Esta reação pode ocorrer em qualquer momento durante a evolução da Hanseníase, sobretudo durante o tratamento específico, que leva à morte bacilar e liberação maciça de antígenos mycobacterianos e conseqüentemente à formação dos imunocomplexos envolvidos na sua fisiopatologia.

No tocante ao grau de incapacidade dos pacientes, no presente estudo $(53 \%)$ dos pacientes evoluíram sem qualquer sinal de incapacidade concordando com o estudo de Gomes e colaboradores 2005 que encontrou $(70,2 \%)$ dos pacientes em condições semelhantes. A maioria dos pacientes também apresentou grau zero de comprometimento, fato que entra em consonância com uma pesquisa feita no extremo sul de Santa Catarina durante os anos 2001 a 2007 em que (50\%) dos pacientes não apresentou incapacidade física (grau zero). A análise desses dados pode sugerir um aumento da busca ativa nas comunidades, estratégias de prevenção e controle da doença mais eficaz, melhora no índice de diagnósticos precoces, maior adesão ao tratamento pelos pacientes e maior acompanhamento desses indivíduos. Porém, não se pode descartar uma subnotificação desses casos.

Dentre os pacientes que evoluíram com algum grau de incapacidade, observou-se predominância do grau I (25,5\%) em comparação as de grau II (21,5\%). Discordando, portanto, do estudo de Pereira e colaboradores 2008 no estado de São Paulo que evidenciou um alto grau de incapacidade em pacientes com Hanseníase onde $(91,6 \%)$ dos participantes foram classificados como grau II. E dos achados de Deepak 2003 e Helene colaboradores 2001 que encontraram maior prevalência de incapacidade grau II em levantamentos internacionais. A análise do grau de incapacidade funcional causado pela Hanseníase é importante para verificar o perfil dos pacientes e a evolução clínica desses. Quanto à caracterização das incapacidades, encontrou-se maior prevalência da perda de sensibilidade (43\%) seguida por presença de atrofias e/ou retrações (18\%). Discordando, também, dos estudos de Pereira e colaboradores 2008 no estado de São Paulo e dos achados de Deepak 2003 e Helene e colabortadores 2001. Sendo estes indicadores, um reflexo do impacto da doença em pacientes sem o devido acompanhamento e que obtiveram um diagnóstico tardio.

A associação entre o grau de incapacidade e o gênero dos pacientes, demonstra através deste estudo que os graus de incapacidade estão mais frequentes no gênero masculino, uma vez que se pode observar que a incapacidade grau I ocorreu em $(32,5 \%)$ nos homens e $(17,4 \%)$ nas mulheres enquanto que a incapacidade grau II prevaleceu com $(25,4 \%)$ no gênero masculino e $(16,3 \%)$ no gênero feminino. Este achado corrobora com determinado estudo (GONÇALVES, MANTELLINI, PADOVANI, 2010), onde os pacientes hansênicos que desenvolveram incapacidades físicas são, em sua maioria, homens, Multibacilares e adultos. Esta correlação também está de acordo com o estudo relacionando a prevalência de complicações por gênero e forma clínica realizado em pacientes diagnosticados com hanseníase, residentes de Palmas-TO por Neves e colaboradores (NEVES et al, 2013), reforçando a relação entre o gênero masculino e a prevalência de incapacidades.

A Classe Operacional Multibacilar apresenta maiores proporções de incapacidades Grau I (31\%) seguida pelo Grau II com (25,9\%), quando comparados com o Paucibacilar Grau I (7,1\%) e Grau II (4,8\%). O que também corrobora com os estudos de estudos apresentados por Gonçalves e colaboradores (GONÇALVES, MANTELLINI, PADOVANI, 2010), onde os pacientes hansênicos que desenvolveram incapacidades físicas são, em sua maioria, Multibacilares.

Em relação à forma de ingresso destes pacientes nesta unidade de referência, este estudo demonstra predominância de pacientes encaminhados pelos serviços de saúde primários (90\%), seguidos por pacientes 
que deram entrada no serviço por demanda espontânea $(6,5 \%)$ e por último através de avaliações de comunicantes $(3,5 \%)$.

A respeito da adesão ao tratamento ocorreu $(99,5 \%)$ de adesão ao tratamento. Este dado sugere a importância dos primeiros contatos do paciente com o serviço de saúde. Uma vez que esse primeiro contato se refere a todo o caminho percorrido pelo paciente que busca atendimento, desde a recepção na unidade básica primária de saúde, a equipe de auxiliares e técnicos de enfermagem até o atendimento médico propriamente dito. Desta forma, tais resultados podem sugerir que o acolhimento adequado realizado pela unidade de referência em estudo, reflete um maior vínculo do paciente com a equipe de saúde o que leva a um aumento da motivação do mesmo para concluir seu tratamento, gerando porcentagens baixas de abandono.

\section{CONCLUSÃO}

O perfil clínico e epidemiológico dos pacientes com hanseníase atendidos pela Unidade de Referência Dr. Marcello Candia em Marituba-PA, é caracterizado por uma predominância do gênero masculino, de 30 a 39 anos, maioria da região metropolitana de Belém, a forma Multibacilar foi predominante. Quanto à classificação de Madrid, foi encontrado, nos pacientes estudados, maior prevalência da forma Dimorfa. A maioria não apresentou incapacidades, foram encaminhados de serviços de saúde e houve uma elevada taxa de adesão ao tratamento. Portanto, conclui-se que o desenvolvimento de ações públicas e educativas para a comunidade e agentes comunitários de saúde, no que diz respeito a divulgação de informações sobre o quadro clinico, diagnostico e tipos de tratamento da Hanseníase se faz fundamental para que ocorra busca ativa dos casos e maior procura desta população aos serviços primários de saúde, gerando o aumento de diagnósticos precoces, além de um segmento adequado da doença e redução dos graus de incapacidade dos pacientes acometidos. Consequentemente ocorrerá aumento da qualidade de vida destes que poderão evoluir sem sequelas após a realização do tratamento adequado para sua respectiva classificação clínica.

\section{REFERÊNCIAS}

1. AZULAY DR. Dermatologia. 6. ed. Rio de Janeiro: Guanabara Koogan; 2017.

2. BRASIL. Ministério da Saúde. Secretaria de Vigilância em Saúde. Departamento de Vigilância Epidemiológica. Baciloscopia em Hanseníase: guia de procedimentos técnicos. Brasília, DF; 2010.

3. BRASIL. Ministerio da Saúde. Guia de controle da Hanseníase. CNDS/CENEPI/FNS/Ministério da Saúde Brasil. Brasília, DF; 2002.

4. Cunha AZS. Hanseníase: aspectos da evolução do diagnóstico, tratamento e controle. Ciência e Saúde coletiva. 2002; 7 (2): 235-242.

5. EIDT LM. Breve história da hanseníase: sua expansão do mundo para as Américas, o Brasil e o Rio Grande do Sul e sua trajetória na saúde pública brasileira. Saúde e Sociedade. 2004, 13(2): 76-88.

6. CUNHA MD, CAVALIERE FAM, HÉRCULES FM, et al. Os indicadores da hanseníase e as estratégias de eliminação da doença em município endêmico do Estado do Rio de Janeiro, Brasil. Caderno de Saúde Pública. 2007; 23 (5): 1187-1197.

7. BRASIL. Ministério da Saúde .2006. Portaria 64/GM de 30 de maio de 2008.

8. ROTTA O. Dermatologia: guia de Medicina ambulatorial e Hospitalar UNIFESP / Escola Paulista de Medicina. São Paulo: Manole; 2008.

9. Brasil. Ministério da Saúde. Hanseníase: Cuidados para evitar complicações. Brasília, DF; 2001.

10. STEFANI MMA. Desafios na era pós genômica para o desenvolvimento de testes laboratoriais para o diagnóstico da hanseníase. Revista da Sociedade Brasileira de Medicina Tropical. 2008; 41(supl 2): 89-94.

11. BRASIL. Ministério da Saúde. 2011. Resolução - RDC № 11 de março de 2011-. ANVISA /MS.

12. BRASIL. Ministério da Saúde. Manual de Prevenção de Incapacidades. Brasília; 2001.

13. AYRES M, AYRES JR M, AYRES DL, et al. BioEstat 5.3: Aplicações Estatísticas nas Áreas das Ciências Biológicas e Médicas. 5. ed. Belém-PA: Publicações Avulsas do Mamirauá; 2007. 
14. LANA FCF, FABRI ACOC, LOPES FN, et al. Deformities due to leprosy in children under fifteen years old as an indicator of quality of leprosy control program in brazilian municipalities. J Trop Med 2013; 2013: 1-6.

15. Andrade M, Bomfim FS. Considerações sobre hanseníase e reações hansênicas. Informe-se em promoção da saúde. 2008; 4(1):13-15.

16. BAKKER M. Epidemiology and Prevention of Leprosy: a cohor study in Indonesia. KIT Publishers; 2005.

17. LIMA LS, JADÃO FRS, FONSECA RNM, et al. Caracterização clínica epidemiológica dos pacientes diagnosticados com hanseníase no município de Caxias, MA. Rev Bras Clin Med, Caxias. 2009; 7: 78-83.

18. AQUINO DMC, ALMEIDA PH. Avaliação do programa de hanseníase do Hospital Universitário Presidente Dutra. São Luís - MA, 1999. Revista da Sociedade Brasileira de Medicina Tropical 33:104105, 2000.

19. WORLD HEALTH ORGANIZATION (WHO). Enhanced global strategy for further reducing the disease burden due to leprosy (plan period: 2011-2015). GENEVA, WHO; 2009.

20. BERNARDES CA, SANTOS AF, PADOVANI CTJ, et al. Incapacidade física em hansenianos de Campo Grande - Mato Grosso do Sul. Hanseníase Internationalis. 2009; 34(1): 17-25.

21. GOMES CCD, PONTES MADA, GONÇALVES HDS, et al. Perfil clínico-epidemiológico dos pacientes diagnosticados com hanseníase em um centro de referência na região nordeste do Brasil. An Bras Dermatol. 2005.

22. LASTÓRIA JC, ABREU MAMM. Hanseníase: diagnóstico e tratamento. Dermatologia. 2012;17(4):173-9.

23. IMBIRIBA EB, HURTADO-GUERRERO JC, GARNELO L, et al V. Perfil epidemiológico da hanseníase em menores de quinze anos de idade, Manaus (AM), 1998-2005. Rev. saúde pública 2008 dez; 42(6):1021-6.

24. BATISTA ES, CAMPOS RX, QUEIROZ RCG, et al. Perfil sócio-demográfico e clínico-epidemiológico dos pacientes diagnosticados com hanseníase em Campos dos Goytacazes, RJ. Rev Soc Bras Clin Med. 2011 abr;9(2):101-6.

25. BARRETO JA, CARVALHO CV, CURY FILHO M, et al. Hanseniase multibacilar com baciloscopia dos esfregaços negativa: a importância de se avaliar todos os critérios antes de se definir a forma clínica. Hansenol Int. 2007;32(1):75-9.

26. SILVA RCP, LOPES A, GUISARD CLMP, et al. História de vida e trabalho de pessoas atingidas pela Hanseníase em Serviços de Saúde do estado de São Paulo. Hansen Int, 2008; 33(1) 9-18.

27. LIENHARDT C, FINE PEM. Type 1 reaction, neurits and disability 15 in leprosy. What is the current epidemiological situation? Leprosy Review 1994; (65): 9-33.

28. MANANDHAR R, LEMASTER JW, ROCHE P. Risk factors for erythe-14 ma nodosum leprosum. International Journal of Leprosy 1999; (67): 270-8.

29. SAUNDERSON P, GEBRE S, BYASS P. Enl reactions and impair -13 ments in the multibacillary cases of the AMFES cohort in central Ethiopia: incidence and risk factors. Leprosy Review 2000; (71): 318-24. 